www.jmscr.igmpublication.org

Impact Factor (SJIF): 6.379

Index Copernicus Value: 71.58

ISSN (e)-2347-176x ISSN (p) 2455-0450

crossref DOI: https://dx.doi.org/10.18535/jmscr/v6i5.161

Journal Of Medical Science And Clinical Research

IGM Publication

An Official Publication of IGM Publication

\title{
Original Research Article \\ Clinical Profile and outcome of Severe Acute Malnutrition (SAM) Children admitted in Nutrition Rehabilitation Centre, City Hospital, Berhampur, Odisha
}

\author{
Authors \\ Bijay Kumar Panigrahi ${ }^{1}$, Sunil Kumar Agarwalla ${ }^{2}$, Nasreen $\mathrm{Ali}^{3}$ \\ ${ }^{1}$ Senior Consultant pediatrics at DHH Koraput and Saheed Laxman Nayak Medical College, Koraput, \\ ${ }^{2}$ Associate Professor, ${ }^{3}$ Junior Resident \\ Department of Pediatrics, M.K.C.G Medical College, Berhampur, Ganjam, Odisha, 760004, India
}

\begin{abstract}
Background: India has one of the highest number of severely malnourished children in the world. It is estimated that around 93.4 lakh children are having severe acute malnutrition (SAM) as per National Family Health Survey (NFHS) 4 and out of this, 10 per cent of SAM with medical complications may require admission to Nutrition rehabilitation centre (NRC). In Odisha, despite laudable progress in reducing child under nutrition between the last two rounds of the NFHS survey (1998-99 and 2005-06; child underweight declining from $54 \%$ to $44 \%$ respectively) and at a faster rate than the national average, there has been little change in SAM children. While no national level survey data have been made available since the NFHS-3 (2005/6), many independent assessments report note that malnutrition levels in the state remain stubbornly high. Severe acute malnutrition (SAM) continues to be an important cause of mortality. Critical care, management of infections, nutritional therapy followed by nutritional rehabilitation is a very important aspect for these children. The present study was, therefore, undertaken to evaluate incidence and clinical profile of patients admitted with severe acute malnutrition in Odisha.

Objective: To assess the clinical profile of SAM among children aged 1-59 months in the NRC of a District Headquater Hospital (DHH), Berhampur

Materials and Method: A hospital based prospective study was carried out from-1-12-2016 to 30.11.2017. A total of 223 children who were admitted to NRC were enrolled in this study and their data was compared and evaluated.

Results: Out of the 223 enrolled in the study, 54.3\% were female.63.4\% were in the age group of 7-24 months.48.4\% belonged to schedule cast.55\% of the children stayed between 7-15 days. The most common co-morbid condition was anemia (94\%) followed by pneumonia or other ARIs (28\%). Out of these $84 \%$ had $z$ score <-3 SD. The recovery rate was $85 \%$. Mean duration of stay was 14 days. Cure rate was $85 \%$.Defaulter rate was $7 \%$.Average weight gain was $12 \mathrm{gms} / \mathrm{kg} /$ day. Follow up rates were $72 \%$.

Conclusions: With cure rate of $85 \%$ and no deaths, NRCs provide live-saving care for children with SAM as demonstrated by the high survival rates of the program. Community-based therapeutic care for children with uncomplicated SAM needs to become a key component of the continuum of care for children with SAM.

Keywords: NRC, Outcome, Severe acute malnutrition.
\end{abstract}




\section{Introduction}

UNICEF estimated malnutrition (45\%) to be the most common cause of under 5 mortality in India and Nigeria accounting for more than $1 / 3$ rd of the deaths. In India prevalence of SAM is $6.4 \%$ in children below 5 years of age with 100 focus districs having high prevalence of malnutrition being situated in 6 states, Bihar, Jharkhand, MP, Rajasthan, Odisha and UP. For proper utilisation of funds and management there is phase wise implementation of NRCs in these areas by UNICEF. It is important to identify the treatment outcome from these NRCs.

Severe acute malnutrition is defined by a very low weight for height (below $-3 \mathrm{z}$ scores of the median WHO growth standards), by visible severe wasting, or by the presence of nutritional oedema $^{[1]}$. Decreasing child mortality and improving maternal health depend heavily on reducing malnutrition, which is responsible, directly or indirectly, for $35 \%$ of deaths among children under five. SAM remains a major killer of children as mortality rates are nine times higher than those in well nourished children.

Although the median under-five case-fatality rate for severe acute malnutrition typically ranges from $30 \%$ to $50 \%$, it can be reduced substantially when physiological and metabolic changes are taken into account. Management of severe acute malnutrition according to WHO guidelines reduced the case-fatality rate by $55 \%$ in hospital settings and recent studies suggest that ready-touse therapeutic foods, can be used to manage severe acute malnutrition in community settings. In order to train health workers in applying this scheme WHO has created a course which, with the aid of institutional partners in Bangladesh, Chile, Gambia, Malawi and the UK, has been conducted in countries in the African, South-East Asia and Western Pacific Regions.

In India, the inpatient model for treatment of severe acute malnutrition (SAM) is still the only model approved at the national level. To date, just a few states have opened up to the communitybased management of acute malnutrition
(CMAM), such as Rajasthan and Odisha. Inpatient facilities to treat SAM children in India are often called Nutrition Rehabilitation Centres (NRCs) or Malnutrition Treatment Centres (MTCs).

Recently, data from a number of different studies shows that this model of treatment has not been performing adequately. It shows that the recovery rates of SAM children attending the inpatient facilities are still low and the defaulter rate is high when compared to the acceptable minimum standard. The recovery rate varies widely in India from state to state and can be as low as $37 \%$. In all the studies, the recovery rate was well below the $75 \%$ standard set by SPHERE. This confirms the well-recognised urgent need to revise and update the model of SAM management in India. ${ }^{[6]}$

\section{Methods}

This is a retrospective data of SAM children admitted to NRC from 1-12-2016 to 30-11-2017 at District headquater hospital, berhampur, Odisha. Institutional ethical clearance was obtained before under taking this study. Data of the children fulfilling the WHO criteria for SAM, between the age group of $1 \mathrm{~m}$ to $59 \mathrm{~m}$, were included in the study.

The WHO criteria to identify SAM in infants more than 6 months and within 59 months of age are, weight-forheight less than -3SD and /or, visible severe wasting and/or, Mid Upper Arm Circumference (MUAC) $<11.5 \mathrm{~cm}$ and/or edema of both feet. The criteria to identify SAM in infants less than 6 months of age are, edema of both feet and/or weight for length less than 3SD(in infants with length more than $45 \mathrm{~cm}$ ) and /or visible severe wasting in infants with less than $45 \mathrm{cms}^{\text {[4] }}$

The following data were collected for the analysis like age, sex, criteria for admission, associated medical complications, type of feeding, immunisation status, response to the treatment, duration of stay in the hospital, extent of weight gain, or weight loss, discharges, discharges against medical advice, readmissions, referrals and death. 
WHO protocol for the management of these SAM children were strictly adhered. The diet F75 and F100 given to these children were prepared in the NRC kitchen. Once stabilized, home based food was started. All the data were analyzed to know the quantitative indicators of the NRC. The quantitative indicators of the NRC are recovery rate, death rate, defaulter rate, weight gain and length of stay. These indicators are also indicative of the outcome of these children admitted to NRC. The objective of the analysis presented here is to assess the effectiveness of NRC in providing therapeutic care for children with SAM in Odisha.

\section{Results}

223 children were fulfilling the criteria for admission to NRC during the study period. Weight for height of all these children were below -3SD. $102(45.7 \%)$ were male and 121(54.3\%) were female. There were $5(2.2 \%)$ of children belonging to the age group of $<6$ months and $139(62.3 \%)$ were between 6-24 months. Children between 25 months-60months were $79(35.4 \%)$ and between 6 months to 24 months were 139 $(62.3 \%)$.[Table 1].The mean duration of stay at NRC was 14 days.12(5.3\%)children stayed for $<7$ days. $123(55 \%)$ children stayed for 7-15days and $88(39.4 \%)$ children stayed for >15days. [Table $2] .206(92.3 \%)$ of the children showed some weight gain during the hospital stay. The average weight gain was $12 \mathrm{gm} / \mathrm{kg} / \mathrm{day} .>15 \%$ weight gain was seen in 191(85.6\%), 15(8.4\%) gained <15\% weight gain.4(1.79\%)children did not show weight gain.13(5.8\%) were defaulters.[Table 5]. Distribution of various complications shows anemia followed by pneumonia, anorexia, diarrhoea, fever, hypoglycemia were major complications associated with SAM [Table 4] with pneumonia (28\%), anorexia 75(33.6\%), diarrhoea 22(9.8\%), anemia 210(94\%) and fever $15(6.7 \%)$. [Table 3]. The quantitative indicators of the NRC were as follows, the recovery rate was $85 \%$, the death rate was $0 \%$, the defaulter rate was $7 \%$, mean weight gain was $12 \mathrm{gm} / \mathrm{kg} / \mathrm{day}$ and mean duration was 14 days [Table6]
Table 1-Age Distribution

\begin{tabular}{|c|c|}
\hline$<6$ Months & $\mathbf{5}(\mathbf{2 . 2 \%})$ \\
\hline 6-24 Months & $\mathbf{1 3 9}(\mathbf{6 2 . 3 \%})$ \\
\hline 25-60 Months & $\mathbf{7 9}(\mathbf{3 5 . 4 \%})$ \\
\hline
\end{tabular}

Table 2-Duration of Stay

\begin{tabular}{|c|c|}
\hline$<7$ days & $\mathbf{1 2}(\mathbf{5 . 3 \%})$ \\
\hline 7-15 days & $\mathbf{1 2 3}(\mathbf{5 5 \%})$ \\
\hline$>15$ days & $\mathbf{8 8}(\mathbf{3 9 . 4 \%})$ \\
\hline
\end{tabular}

Table 3-Major Complications of SAM

\begin{tabular}{|l|c|}
\hline Pneumonia & $\mathbf{6 3}(\mathbf{2 8 \%})$ \\
\hline Anorexia & $\mathbf{7 5 ( 3 3 . 6 \% )}$ \\
\hline Diarrhoea & $\mathbf{2 2}(\mathbf{9 . 8 \%})$ \\
\hline Anemia (mild, moderate and severe) & $\mathbf{2 1 0}(\mathbf{9 4 \%})$ \\
\hline Fever & $\mathbf{1 5}(\mathbf{6 . 7 \%})$ \\
\hline
\end{tabular}

Table 4-Complications Associated with SAM

\begin{tabular}{|l|c|}
\hline Pneumonia,ARI and LRTI & $\mathbf{6 3}$ \\
\hline Anorexia & $\mathbf{7 5}$ \\
\hline Diarrhoea & $\mathbf{2 2}$ \\
\hline Fever & $\mathbf{1 5}$ \\
\hline Severe Anemia & $\mathbf{1 0}$ \\
\hline Hypoglycemia & $\mathbf{4}$ \\
\hline Dehydration & $\mathbf{2}$ \\
\hline Jaundice & $\mathbf{1}$ \\
\hline Vomiting & $\mathbf{2}$ \\
\hline Skin Lesions(dermatitis,ulcer) & $\mathbf{6}$ \\
\hline Rickets & $\mathbf{1}$ \\
\hline AOM & $\mathbf{3}$ \\
\hline Cerebral Palsy & $\mathbf{7}$ \\
\hline Global Developmental Delay & $\mathbf{2}$ \\
\hline UTI & $\mathbf{3}$ \\
\hline Congenital Heart Disease & $\mathbf{4}$ \\
\hline Congenital Cataract & $\mathbf{1}$ \\
\hline Otitis & $\mathbf{1}$ \\
\hline Monoparesis & $\mathbf{1}$ \\
\hline Total & $\mathbf{2 2 3}$ \\
\hline
\end{tabular}

Table 5-outcome

\begin{tabular}{|l|c|}
\hline Discharge with $>15 \%$ Weight gain & $\mathbf{1 9 1}(\mathbf{8 5 . 6 \%})$ \\
\hline Discharge with $<15 \%$ Weight gain & $\mathbf{1 5 ( 8 . 4 \% )}$ \\
\hline Non responders & $\mathbf{4 ( 1 . 7 \% )}$ \\
\hline Defaulters & $\mathbf{1 3 ( 5 . 8 \% )}$ \\
\hline Death & $\mathbf{0}$ \\
\hline
\end{tabular}

Table 6-Outcome Indicators of NRC

\begin{tabular}{|l|l|}
\hline Recovery Rate & $\mathbf{8 5 \%}$ \\
\hline Death Rate & $\mathbf{0}$ \\
\hline Defaulter Rate & $\mathbf{5 . 8 \%}$ \\
\hline Weight Gain (mean SD in gms/kg/day) & $\mathbf{1 2}$ \\
\hline Length of stay (mean SD in days) & $\mathbf{1 4}$ \\
\hline Bed occupancy rate & $\mathbf{8 4 \%}$ \\
\hline Follow up rate & $\mathbf{7 2 \%}$ \\
\hline
\end{tabular}




\section{Discussion}

NRC is a place where SAM children are managed methodically and scientifically. NRC attached to a tertiary level hospital has the added benefit of proper management of complicated SAM like shock and sepsis. ${ }^{[5]}$ Many of our children got discharged when once the complications were under control. Acute respiratory tract infections and Acute Gastroenteritis were the common associated complications in SAM children$33.36 \%$ (85) of pneumonia and $18.89 \%$ (48) of diarrhoea cases in Bharathi et al similar findings were found in our study. ${ }^{[2]}$ Anaemia was the other common complication, $94 \%$ in our study when compared to $20.1 \%$ in other studies. ${ }^{[6]}$ Our NRC achieved good outcome with respect to recovery rate of $85 \%$, Death rate of $0 \%$ and $5.8 \%$ defaulter rate is acceptable according to the WHO protocol. Critically ill patients were reffered to nearby MKCG Medical College for ICU care, this amounted to zero death in our study.

The recovery rate in our study was good, $81 \%$ as against other studies where it was $33.6 \%$ in Mahama Saaka et al and $66 \%$ in Maurya et al. ${ }^{[3,7]}$. The mean weight gain which was similar in our study when compared to other studies$14 \mathrm{gm} / \mathrm{kg} /$ day. ${ }^{[8]}$ The defaulter rate was better in our study, $5.8 \%$, when compared to $53 \%$ in the Mahama et al study. ${ }^{[3]}$ Limitation of our study was inadequate follow up. Hence, Hospital based management of these children in specialised feeding centre is very important for faster recovery and a better weight gain. Thus improvement in nutritional status is necessary in the severely malnourished children at NRCs to have a better outcome. ${ }^{[9]}$

\section{Conclusion}

With cure rate of $85 \%$ and no deaths, NRCs provide live-saving care for children with SAM as demonstrated by the high survival rates of the program. A community based program for the management of SAM without complications and for those who are to be followed up after NRC care should be in place to complement the services of NRC. Moreover, each child should be line listed and tracked in the community before and after discharge using the available health care/ICDS\ASHA workers and the system of mother child tracking system(MCTS)/mobile phone SMS. Research and development consensus on locally prepared therapeutic food proxies as used in the regional NRCs should be considered as a national priority.

\section{Reference}

1. Ministry of Health and Family Welfare Government of India, Operational guideline: facility based management of SAM children, Module. 2011:4-6. Available from: http://www.rajswasthya.nic.in/MTC\%20G uideline- \%20MOHFW.pdf

2. Bharathi S, Anuradha K, Rao JV. An experience at a tertiary level hospital NRC in management of severe acute malnutrition in children aged between 6-59 months adopting World Health Organization recommendations. Res Health Sci. 2016;1(1):41-50.

3. Saaka M, Osman SM, Amponsem A, Ziem JB, Abdul-Mumin A, Akanbong $\mathrm{P}$ et al. Treatment outcome of severe acute malnutrition cases at the tamale teaching hospital. J Nutrition Metabol. 2015;2015:1-8.

4. Singh K, Badgaiyan N, Ranjan A, Dixit HO, Kaushik A, Kushwaha KP et al. Management of children with severe acute malnutrition: experience of nutritional rehabilitation centres in Uttar Pradesh, India. Indian Pediatr. 2014;51:21-5.

5. Hossain MI, Dodd NS, Ahmed T, Miah GM, Jamil KM, Nahar B et al. Experience in managing severe acute malnutrition in a government tertiary treatment facility in Bangladesh. J Health Popul Nutr. 2009;27(1):72-9.

6. Mathur A, Tahilramani G, Yadav D, Devgan V. Experience in managing 
children with severe acute malnutrition in nutrition rehabilitation centre of tertiary level facility, Delhi, India. Int J Contemp Pediatr. 2016;3(2):597-600.

7. Maurya M, Singh DK, Rai R, Mishra PC, Srivastava A. An experience of facility based management of severe acute malnutrition in children aged 6-59 months adopting the World Health Organization recommendations. Indian Pediatr. 2014;51:481-3.

8. Teferi E, Lera M, Sita S, Bogale Z, Datiko DG, Yassin MA. Treatment outcome of children with severe acute malnutrition admitted to therapeutic feeding centres in Southern Region of Ethiopia. Ethiopia J Health Develop. 2014;24:234-8.

9. Nagar RP, Nagar T, Gupta BD. Treatment outcome in patients with severe acute malnutrition managed with protocolised care at malnutrition treatment corner in Rajasthan, India: a prospective observational study (quasi-experimental). Int J Res Medical Sci. 2016;4(1):238-45. 